\title{
Safety of Cargo Aircraft Handling Procedure
}

\author{
Daniel Hlavaty ${ }^{1}$, Jakub Kraus ${ }^{1 *}$
}

\author{
${ }^{1}$ Department of Air Transport, Faculty of Transportation Sciences, Czech Technical University in Prague, Prague, \\ Czech Republic \\ *Corresponding author: Czech Technical University in Prague, Faculty of Transportation Sciences, Department of Air \\ Transport, Horska 3, 12800 Prague, Czech Republic, Email: kraus@fd.cvut.cz
}

\begin{abstract}
The aim of this paper is to get acquainted with the ways how to improve the safety management system during cargo aircraft handling. The first chapter is dedicated to general information about air cargo transportation. This includes the history or types of cargo aircraft handling, but also the means of handling. The second part is focused on detailed description of cargo aircraft handling, including a description of activities that are performed before and after handling. The following part of this paper covers a theoretical interpretation of safety, safety indicators and legislative provisions related to the safety of cargo aircraft handling. The fourth part of this paper analyzes the fault trees of events which might occur during handling. The factors found by this analysis are compared with safety reports of FedEx. Based on the comparison, there is a proposal on how to improve the safety management in this transportation company.
\end{abstract}

Keywords

Air cargo transportation - FedEx - FTA - Handling - Predictive Analysis - Safety — Safety factors

\section{Introduction}

People were trying to find ways how to fly from the beginning of 20th century. In 1912, the brothers Wrights finally succeeded. Since then the aviation has gone through a large development. As people started travelling by planes, the need for transportation of freight has consequently increased and first cargo transport companies were found. In the second half of the 20th century, Federal Express (now FedEx) was founded. This company was focused solely on air freight express, not for the transport of people.

One of the most important parts of air transport operation is safety. The level of safety in air traffic companies is indicated by a number of air traffic incidents and accidents [1]. This number of incidents and accident is currently so low that the air traffic companies have to analyse smaller events or minor indicators instead of real accidents, which happen rarely
[2]. These events are monitored and analysed on a daily basis. This paper evaluates current quality of safety during the cargo handling of FedEx aircrafts and search for additional ways how to improve the safety procedures and safety performance $[3,4]$.

\section{Specifics of air cargo transportation}

Transportation of freight is different from transportation of passengers, especially the procedures of handling are different. Actions which have to be performed during technical handling of passengers aircraft are, inter alia, boarding of passengers, loading and unloading of baggage, loading and unloading of cargo, catering supplies and cleaning of aircraft. On the other hand, activities performed during business handling of cargo aircrafts can include only crew boarding and loading and unloading of cargo. 
In cargo transportation business there are three ways of transporting cargo. The first method of cargo transportation is bulk. Bulk aircrafts are divided into several segments which are loaded with packages. These packages have to be loaded one after another and secured by correct ways of loading. The correct procedure for loading packages at FedEx is called T-stacking. T-stacking prevents packages from crash and ensures uniform weight distribution in each segment of aircraft. A pattern of T-stacking loading is displayed on figure 1 below.

Freight loaded into the unit load devices (ULD) represents the second way how to transport freight by air. There are two types of unit load devises. One of them is a container. A metallic container can have many shapes. Smaller containers are used for loading on the main deck of narrow-body aircrafts or loading on the lower deck of these aircrafts. Larger containers are used for loading on main and lower decks of wide-body aircrafts. Some containers are specially modified to transport vehicles or cattle.

The second type of unit load devices is a pallet. Freight is loaded into pallets, but individual packages have to be secured against crash by safety nets. Pallets do not have walls like containers so freight cannot lean on. The function of walls is provided by safety nets.

All of ULDs (containers, pallets and nets) have to be certificated according to the IATA rules. IATA has published ULD Regulations, which contain all rules for construction, transportation and using of unit load devices. ULD Regulations also define the method of marking. Every ULD has its own unique designation. It is composed of prefix (type of container), unique number and suffix (code of airline) [5].

The last way how to transport freight by air is the use of special aircrafts to transport oversized and extremely heavy freight. An example of that aircraft can be Antonov An-225 Mrija.

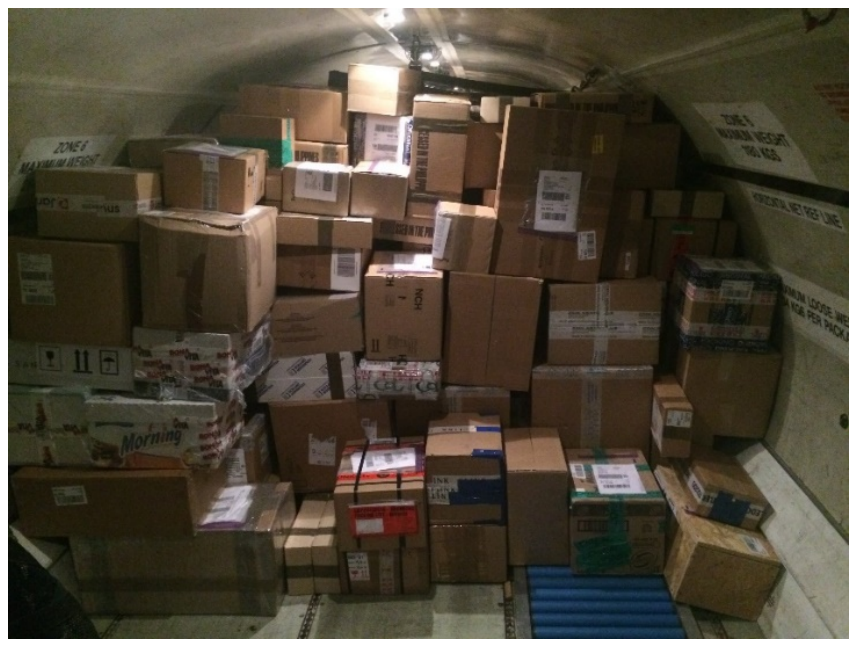

Figure 1. Bulk loading

\section{Procedures of Handling}

Aircrafts that are used for distribution of shipments to areas like the Czech Republic are called Feeders in FedEx terminology. Aircrafts used for this distribution are mostly ATR types.

The FedEx operation at Vaclav Havel Airport Prague can be divided into the AM operation (arrivals) and PM operation (departures). FedEx ATR aircraft arrives on Tuesday, Wednesday, Thursday and Friday. Departures are on Monday, Tuesday, Wednesday and Thursday. During the day or on non-flying days is the plane parked on apron E1A. Handling is provided by Menzies Aviation Company.

\subsection{Unloading}

The unloading process usually starts few minutes after the aircraft arrives. Several types of ground support equipment (GSE) are used for unloading. When the propeller is stopped and anti-collision beacon is switched off, the loading leader can connect a ground power unit (GPU). Loading belts are used for unloading shipments from aircraft. Shipments from loading belts are moved to trolleys (the same trolleys, which are used to transfer baggage). These trolleys are moved to a warehouse where the sorting is performed. It is very important to follow few safety rules during unloading and during approaching of GSE to aircraft. The most important rule is using a guide person. The guide person is a person who navigates GSEs driver and shows by hand signals the distance between the GSE and the aircraft. The minimum distance between the loading belt and the edge of cargo doors must be longer than 5 feet. The second important rule is testing of potential defects of each ground support equipment before final approach of the aircraft. Other safety rules can include e.g. correct positioning of cones, chocking wheels or walking around wings and propeller. All the safety rules are supervised by a Ramp Agent of FedEx.

FedExs Ramp Agents is determined to ensure compliance of all safety rules. His further responsibility is preventing the delays of handling. Every small delay has to be notified to the FedEx operation centre in Paris. The Ramp Agents have to fill a special safety report during every morning and evening operation. This report includes fields to fill in exact time when each handling activity was executed.

\subsection{Loding}

Every shipment has to be prepared before loading into aircraft. This preparation includes $\mathrm{x}$-ray and weighting of every shipment. After that, shipments are loaded to vans and transferred to airports warehouse. After that, the shipments are put over from vans to trolleys. Trolleys are weighted by fork-lift weight and the weight of shipments is calculated by difference of gross weight and weight of empty trolley. A table with these weights is sent to the Load Control office, where a Load Plan is drawn up.

Before any loading is started, it is important to check that tail stand is fixed on the aircrafts tail. A tail stand secures 
the construction of an aircraft in case of overturning to tail. It is also important to make sure that cones are in a correct position and that aircraft is fully choked.

Any loading can be started only in accordance to the Load Plan which is created by the Load Control office. Loading usually takes one hour. All the loading activities are checked by FedExs Ramp Agent. Every safety deviation is written down to a form report. After a departure, all deviations are reported to the Head office of handling company. This reporting ensures appropriate quality of handling services.

\section{Identification and rating of risks}

European Aviation Safety Agency (EASA) publishes all the safety regulations in civil aviation in all European air space. Regulations are also set for handling procedures. The two most important ones are Regulation (EU) No 376/2014 [6] and Commission Implementing Regulation (EU) 2015/1018 [7], where the second one defines risky events that can happen during all activities related to air civic aviation. These events are classified into logical groups. Regulation 2015/1018 defines events, which are used in safety reporting systems like SMS (Safety Management System) for easier classification of reported events.

Potential high-level events that can happen during a handling procedure of FedExs aircraft, or any other cargo aircraft, have been selected from EASA classification (selected from [7]):

- A collision or a near collision on the ground or in the air, with another aircraft, terrain or obstacle.

- Leakage of any fluid which resulted in a fire hazard or possible hazardous contamination of aircraft structure, systems or equipment, or which has or could have endangered the aircraft, its occupants or any other person.

- Push-back, power-back or taxi interference by vehicle, equipment or person.

- Foreign object on the aerodrome movement area which has or could have endangered the aircraft, its occupants or any other person.

- Passengers or unauthorized person left unsupervised on apron.

- Aerodrome security related occurrences (for example: unlawful entry, sabotage, bomb threat).

- Incorrect handling or loading of passengers, baggage, mail or cargo, likely to have a significant effect on aircraft mass and/or balance (including significant errors in loadsheet calculations).

- Incorrect stowage or securing of baggage, mail or cargo likely in any way to endanger the aircraft, its equipment or occupants or to impede emergency evacuation.

- Transport, attempted transport or handling of dangerous goods which resulted or could have resulted in the safety of the operation being endangered or led to an unsafe condition (for example: dangerous goods inci- dent or accident as defined in the ICAO Technical Instructions).

- Non-compliance with required aircraft ground handling and servicing procedures, especially in de-icing, refuelling or loading procedures, including incorrect positioning or removal of equipment.

- Failure, malfunction or defect of ground equipment used for ground handling, resulting into damage or potential damage to the aircraft (for example: tow bar or GPU (Ground Power Unit)).

- Missing, incorrect or inadequate de-icing/anti-icing treatment.

- Damage to aircraft by ground handling equipment or vehicles including previously unreported damage.

- Any occurrence where the human performance has directly contributed to or could have contributed to an accident or a serious incident.

A Fault tree analysis (FTA) has been selected to find safety factors. For the application of FTA, events specified in [7] had to be detailed because the FTA requires welldescribed top events $[8,9]$. As stated, there can be high numbers of detailed events, i.e. many events can be classified under the points from [7].

\subsection{Fault Tree Analysis}

Fault tree analysis (FTA) was invented with the aim of searching for original reasons of accident in civil aviation and nuclear power engineering. It is based on quality definition of top events, which means that the top events have to be as detailed as possible. Also, an important part of the FTA is determination of the circuit of investigation. Results of the FTA are represented by factors that could be the first in a sequence of events, which lead into an accident. FTA is based on a graphical representation. Such graphical representation helps finding the real factors [7].

FTA is shown on one specific detailed event Damage of propeller due to collision of propeller and trolley, which can be classified under Damage to aircraft by ground handling equipment or vehicles including previously unreported damage [7].

\subsection{Damage of Propeller due to Collision of Pro- peller and Trolley}

Any collision occurring during handling means high financial expenses. A collision between trolleys and propeller is very probable, because trolleys are being manipulated in a close proximity to propeller. Too close approaching of trolleys to propeller is prevented by positioning cones. Cones have to be located one meter from the planes nose, ends of wings and tail. Cones have to be located also before front and rear loading doors and in front of propeller.

FedEx focuses on two main rules walking around propeller and wings and using a guide person. Prohibition of walking or riding with GSE under wings is not a standard rule in other airlines. It causes that loaders with experience 


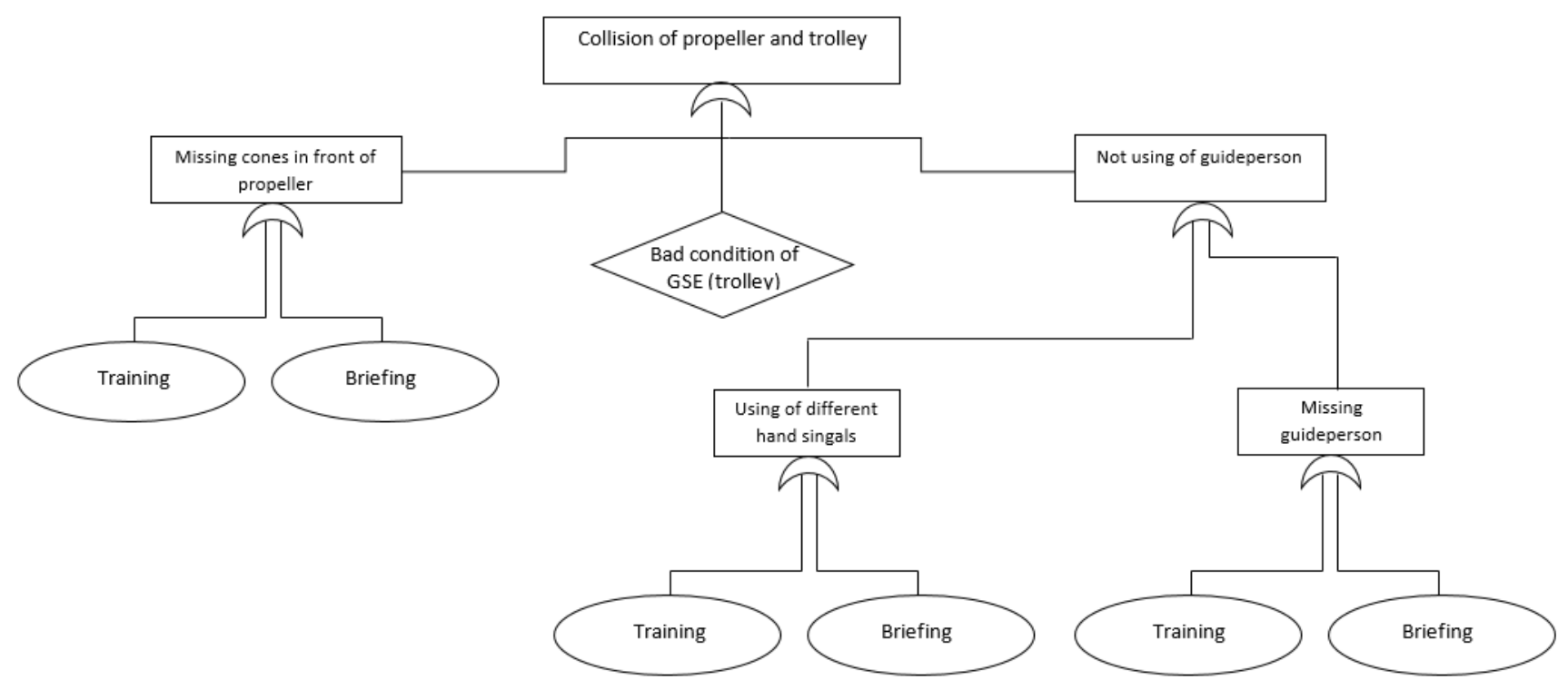

Figure 2. FTA - Collision of propeller and trolley

from other airlines can forget about the prohibition of walking under wings during FedEx operation. The Team leader of loading should therefore notify the loaders of every specific FedEx rule before loading or unloading gets started. The first factor found by FTA is the absence of briefing before operation. The second factor could be training of loaders, which should know all FedExs rules from the trainings they have to pass.

All leaders should also know where to place cones around aircraft. If they did not pass training on the handling procedures of FedExs aircraft and would not perform proper briefing, then the cones could end up in wrong position, which might have a negative impact on safety distance between trolleys and propeller.

Another important rule during FedEx operation is using of guide person. If a guide person is not used or if it uses incorrect hand signals, the safety distance might not be kept and collision can happen. Without a proper training and briefing before loading, a loader would not know the right hand signals or procedures that every guide person has to use during each approach of GSE to the aircraft.

The last factor which has been found by the FTA is bad GSE condition. However, such factor like a bad condition of GSE, are beyond the influence of FedEx. FedEx assumes that the condition of GSE is good, because all GSE are owned by handling company Menzies Aviation.

Figure 2 shows a preview of FTA tree, which considers factors like training, absence of briefing or bad GSE condition.

\section{Determination of factors}

FTA has been applied on all defined top events chosen from EASA classification list that are specific for FedEx opera- tion. Results of this analysis are presented on a list of factors, which are found to be the original causes of accidents or incidents. The way how to find out if a current collection of safety data in FedEx is focused on collection of real factors is comparison. FedEx has a wide database of morning and evening reports in which all safety deviations that happened since 2011 can be found. The List 1 below shows several kinds of safety deviations and its quantity. All data in the List 1 have been gathered by analysing each report since 2011. The List 2 below shows all factors, which have been found by the FTA. A comparison of these two lists enables us to assess if the surveillance of FedExs Ramp Agents is really focused on the original factors or if it focuses only on smaller events. Effective prevention against accidents during handling can occur only if both surveillance and safety data collection are focused on correct original factors.

List 1: Quantity of safety deviations from 2011 to 2016

- Wrong weight written on trolleys

- Walking under wings

- Wrong positioning of cones

- Issues with warehouse messages

- Chocking

- Missing of guide person

List 1: Factors found by the FTA

- Training

- Briefing before the start of handling

- FOD check

After comparing the List 1 and 2, we can conclude that the factors found by the FTA do not match with the events in FedEx reports. It means that the reports have to be changed 
in order to increase the quality and usability of safety data collection.

\section{Conclusion}

Safety management is an important part of every airline. The aim of safety management is prevention from accidents and incidents in operations. Primary objective of the safety management work is a research of official reports of incidents and accidents. Nevertheless, this is currently not enough [10]. Today, it is important to perform a surveillance of events and factors and thoroughly collect this data.

Two types of analysis and their following comparison has enabled us to found out that current safety data collection supplemented by the reports filled by a Ramp Agent are not sufficient enough. It is necessary to change these reports.

The first problem with the current reports is a lack of their focus. The reports are not focused on real problems, which can lead to accidents. It is necessary to add some fields into current reports. These new fields would focus on safety factors. Then, after some time, a good database of factors deviations will be created. A thorough analysis of this database will help us finding which factors are repeated most often. Consequently, FedEx will be able to improve its handling procedures to prevent the occurrence of undesirable factors during operations. As a result, the overall safety will be increased.

The second problem with the current reports is the way how data is currently collected. For example, the reports miss checkbox fields. Based on FedExs reports analysis it was found that several important reported events in reports do not have its own field. These events are currently filled into a remarks field so the recording of important events happens only if the Ramp Agent does not forget to fill this field and/or if he has enough time for that during handling. Existence of checkboxes on reports could have a positive impact on quantity of collected safety data.

Only a focus on the real causes of accidents or incidents and ways how the data about such incidents is collected can improve the risk management in FedEx Czech Republic.

\section{Acknowledgments}

This paper was supported by the Grant Agency of the Czech Technical University in Prague, grants No. SGS17/154/OHK2/2T/16 and SGS17/152/OHK2/2T/16.

\section{References}

[1] V. Plos et al. The role of risk-based indicators for the air carrier. In Proceedings of 20th International Conference Transport Means 2016, pages 213-215. Kaunas University of Technology, 2016.

[2] Vladimir Plos, Peter Vittek, and Karel Jerabek. Reports about occurrence of events with effect on aviation safety.
MAD - Magazine of Aviation Development, 2(10):13-16, 2014. doi: 10.14311/mad.2014.10.04. URL https : //doi.org/10.14311/mad.2014.10.04.

[3] A. Lalis et al. Predicting safety performance to control risk in military systems. In Proceedings of the 2017 International Conference on Military Technologies (ICMT), pages 392-396. University of Defence, 2017.

[4] Andrej Lalis and Peter Vittek. Safety KPIs - monitoring of safety performance. MAD - Magazine of Aviation Development, 2(11):9-12, sep 2014. doi: 10.14311/mad. 2014.11.02. URL https://doi.org/10.14311/ mad.2014.11.02.

[5] VRR Aviation. Identifying a Unit Load Device (ULD), 2017. URL http: / /vrr-aviation.com/ uld-info/uld-id-code/. [Online].

[6] Regulation (EU) No 376/2014, 2014. URL http: / / eur-lex.europa.eu/legal-content/EN/ TXT / ?uri=CELEX: 32014R0376. [Online].

[7] EU Commission Implementing Regulation (EU) No 2015/1018, 2015. URL http: / / eur-lex. europa. eu/legal-content/EN/TXT/ ?uri=CELEX: 32015R1018. [Online].

[8] Ales Bernatik. Prevence zavaznych havarii I [Prevention of major accidents]. Sdruzeni pozarniho a bezpecnostniho inzenyrstvi, Ostrava, 2006. ISBN 80-86634-89-2. (In Czech).

[9] Jana Ondraskova and Jakub Kraus. Analysis of appropriate methods for assessment of safety in aviation. MAD - Magazine of Aviation Development, 2(11):4-8, sep 2014. doi: 10.14311/mad.2014.11.01. URL https : //doi.org/10.14311/mad.2014.11.01.

[10] Peter Vittek, Andrej Lalis, Slobodan Stojic, and Vladimir Plos. Challenges of implementation and practical deployment of aviation safety knowledge management software. In Communications in Computer and Information Science, pages 316-327. Springer International Publishing, 2016. doi: 10.1007/ 978-3-319-45880-9_24. URL https://doi.org/ $10.1007 / 978-3-319-45880-9 \_24$. 